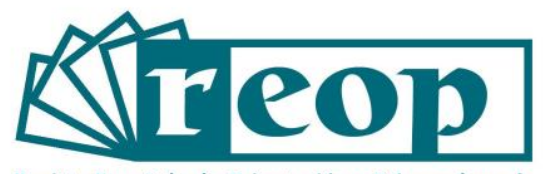

Revista Española de Orientación y Psicopedagogía

\title{
ANÁLISIS DE VALIDEZ DE CONSTRUCTO Y CONFIABILIDAD DE DOS INSTRUMENTOS PARA EVALUAR LAS ACTIVIDADES DE ORIENTACIÓN PROFESIOGRÁFICA
}

\section{ANAL YSIS OF CONSTRUCT VALIDITY AND RELIABILITY OF TWO INSTRUMENTS TO EVALUATE PROFESSIONAL ORIENTED ACTIVITIES}

\author{
Walter A. Mata-López ${ }^{1}$ \\ Universidad de Colima. Facultad de Ingeniería Mecánica y Eléctrica. Colima, México \\ Luis G. Juárez-Hernández \\ Centro Universitario CIFE². Cuernavaca, México \\ Sergio Tobón \\ Centro Universitario CIFE. Cuernavaca, México \\ Osval A. Montesinos-López \\ Universidad de Colima. Facultad de Telemática. Colima, México
}

\section{RESUMEN}

Se realizó un análisis de la validez de constructo y confiabilidad de un cuestionario para evaluar una jornada profesiográfica y una rúbrica para evaluar la exposición de las carreras. El análisis de validez de constructo se efectuó mediante un análisis factorial exploratorio y la confiabilidad mediante el Alfa de Cronbach. Para el cuestionario, el análisis mostró que solo el $53 \%$ de sus ítems presentaron cargas factoriales significativas (CF $>0.65)$, los cuales estuvieron representados en tres dimensiones (Grado de satisfacción con las características de la carrera, Grado de

\footnotetext{
${ }^{1}$ Correspondencia: Walter A. Mata López. Correo-e: wmata@ucol.mx, web: https://www.ucol.mx

2 Ciencia e Innovación para la Formación y el Emprendimiento.
} 
satisfacción con la facultad y Recomendación de la FIME $^{3}$ y de la carrera) de las cinco que se propusieron de manera inicial. Para la rúbrica, los resultados se correspondieron con lo propuesto teóricamente. La confiabilidad de ambos instrumentos fue adecuada (Alfa de Cronbach de 0.907 y 0.908). Se puede concluir que el análisis efectuado favoreció la depuración de los instrumentos; sin embargo, la limitación del presente estudio fue el tamaño de muestra por lo que los resultados no pueden ser considerados concluyentes. Se aportan implicaciones del estudio para la orientación profesional.

Palabras clave: orientación profesional, elección de profesión, validez, fiabilidad, análisis factorial.

\section{ABSTRACT}

An analysis of the construct validity and reliability of a questionnaire to evaluate a professions day, as well as a rubric to evaluate the career exhibitions were carried out. The analysis of construct validity was carried out by an exploratory factor analysis and reliability through Cronbach's alpha. The questionnaire showed that only $53 \%$ of its items had significant factorial loads $(C F>0.65)$, which were represented in three dimensions (Degree of satisfaction with the characteristics of the career, Degree of satisfaction with the faculty and Recommendation of FIME and the career) of the five we initially proposed. For the rubric, the results corresponded to what we theoretically proposed. The reliability of both instruments was adequate (Cronbach's Alpha: 0.907 and 0.908 ). We can conclude that the analysis allowed us to refine and improve the instruments; however, the limitation of the present study relates to the sample size, leading to results that cannot be considered as conclusive ones. Implications of the study for career guidance are provided.

Key Words: career guidance, career choice, validity, reliability, factor analysis.

\section{Cómo citar este artículo:}

Mata-López, W.A., Juárez-Hernández, L.G., Tobón, S. y Montesinos-López, O.A. (2021). Análisis de validez de constructo y confiabilidad de dos instrumentos para evaluar las actividades de orientación profesiográfica. Revista Española de Orientación y Psicopedagogía, 32(2), 47-68. https://doi.org/10.5944/reop.vol.32.num.2.2021.31278

\section{Introducción}

La matrícula de estudiantes en educación superior es uno de los factores importantes que consideran las universidades que ofrecen carreras, puesto que, al pretender satisfacer necesidades sociales, económicas y culturales, un descenso en la matrícula puede traer consigo problemas en cascada que podrían poner en riesgo la pertinencia de dicha oferta educativa (Zafra-Tristancho et al., 2014; González, 2015; Barbón-Pérez y Fernández-Pino, 2018). Por lo tanto, la promoción de la oferta educativa y cómo se realiza esta es un aspecto significativo o de

${ }^{3}$ Facultad de Ingeniería Mecánica y Eléctrica 
interés. En este sentido, la forma en que se promocionan es altamente variada y se establecen distintas estrategias para ello como, por ejemplo: cursos, talleres, publicidad en distintos medios, redes sociales, entre otras (Arroyo-Hernández et al., 2012; Brito et al., 2015; Zapata y Tejeda, 2016; Canek et al., 2019).

Lo anterior es importante ya que si bien existe un gran número de factores intrínsecos (personalidad, habilidades, expectativas y percepciones), y extrínsecos (estatus socioeconómico, antecedentes culturales, currículum escolar, actitudes y valores de profesores y padres) (BravoTorres y Vergara-Tamayo, 2018; Mata-López y Tobón, 2019), que hacen a los estudiantes de preparatoria elegir una carrera, es fundamental implementar estrategias para que las universidades proporcionen información oportuna de las carreras que ofrecen, para que el estudiante pueda hacer su elección de una manera adecuada (Díaz-lzaguirre et al., 2019; Romero-Rodríguez et al., 2019).

En este sentido, Villada et al. (2002) indican que el alumno no cuenta con el criterio suficiente para tomar una decisión acertada, por lo tanto, si se realiza una mala elección de carrera regularmente puede determinar deserción por reprobación o abandono (Fuentes-Navarro, 2010). A este respecto, es también importante considerar que algunas veces los estudiantes se ven obligados a elegir una carrera de segunda o tercera opción afín o no a su área de interés debido a los criterios de selección de las universidades. Este aspecto reafirma la importancia de que el alumno o estudiante además de contar con la información de la carrera de interés, posea información pertinente y objetiva de opciones alternas. Una adecuada elección de carrera o bien de una segunda opción resulta fundamental para que el estudiante logre graduarse (Lara-Barrón y Valadez-Díaz, 2011).

Como puede observarse, la elección de carrera responde a múltiples factores, por lo que las universidades deben implementar estrategias de promoción y orientación profesional (HernándezSalamanca, 2020) para que los estudiantes tengan información para identificar la carrera qué más les interese, así como las distintas alternativas afines (Lara-Barrón y Valadez-Díaz, 2011; ÁlvarezLires et al., 2014; Quatrocchi et al., 2017). Acorde a lo anterior, deben establecerse estrategias de orientación vocacional previas al ingreso a la educación superior para que los estudiantes tengan una mayor certidumbre de la carrera que elegirán (Aguilar-Salinas et al., 2018); además, puede permitir que los estudiantes muevan sus intereses a medida que adquieran más conocimientos acerca de la carrera (Manzano-Sánchez et al., 2019). Es importante reconocer que contar con alumnos que tengan suficiente información de las carreras de su interés y que mediante esta realicen una adecuada elección, beneficia a la institución educativa ya que se reducen las tasas de reprobación y deserción.

Una de las áreas de mayor conflicto por una mala elección de carrera, o bien por la baja matrícula es el área de ingenierías en general. La globalización y el desarrollo tecnológico sin precedentes, han incrementado la alta demanda de profesionales de la ingeniería (Aničić et al., 2017), demanda que puede satisfacerse si se conocen las necesidades del mercado laboral en cuanto a la serie de conocimientos y habilidades que necesitan los graduados, por lo que dar a conocer la oferta académica de las universidades es una excelente estrategia para lograr incrementar la matrícula en carreras consideradas como duras (Mata-López y Tobón, 2019).

En la literatura se encuentran algunos antecedentes que tienen como propósito general el incremento de la matrícula de carreras relacionadas con la computación, de acuerdo con una serie de estrategias. En este orden, de las de mayor relevancia se reportan campamentos (Rodríguez et al., 2014); talleres (Byrne et al, 2017); proyectos con temas de actualidad (Zaharakis et al., 2016); comunidades de aprendizaje (Payton et al., 2016); desarrollo de actividades extracurriculares (De Vega et al., 2017); jornadas, muestras o ferias profesiográficas (Arroyo-Almaguer et al., 2019); exposiciones o presentaciones de carreras (Durán, 2014; Retana-Alvarado y Vázquez-Bernal, 2016; Mata-López et al., 2019), entre otras. 
A este respecto, se hace énfasis en las jornadas profesiográficas (JP) y exposiciones de carreras (EC) que se llevan a cabo en la Universidad de Colima (UCol). El objetivo de estas actividades es el de dar a conocer información detallada de las carreras para atraer estudiantes a que se matriculen en alguna de ellas. Las JP se programan en cada ciclo de ingreso, realizándose en cada una de las facultades de los campus de la universidad. Las actividades que se realizan en estas jornadas consisten en recorridos por todas las instalaciones con su respectiva presentación (salones de clase, laboratorios, talleres, áreas comunes, cubículos de profesores, etc.), y además se exhiben proyectos de estudiantes de las distintas carreras que se ofrecen. Por su parte, las EC se realizan durante las JP, pero principalmente esta actividad se lleva a cabo en las instalaciones propias de los bachilleratos previa programación; en ella se presentan las carreras que ofrecen las facultades, perfil del estudiante, del egresado, actividades que desarrolla, ámbito laboral, instalaciones, proyectos, y becas principalmente. Estos eventos tienen cuatro años celebrándose, sin embargo, hasta el momento todas estas actividades no se habían evaluado a detalle, por lo que no se podía conocer el impacto tanto en el grado de satisfacción con las actividades desarrolladas como en la percepción de estas por parte de los asistentes. Razón por lo que es importante establecer mecanismos de evaluación de las estrategias de promoción para verificar la significancia para la institución y los posibles estudiantes, permitiendo de este modo, estar en condiciones de proponer mejoras para aplicarlas en periodos posteriores (Mata-López y Tobón, 2019).

Por lo anterior, Mata-López et al. (2019), diseñaron instrumentos para la "Encuesta de la Jornada Profesiográfica" y la "Rúbrica de la Exposición de la Carrera de Ingeniería en Sistemas Computacionales". La encuesta permite evaluar los intereses personales, grado de satisfacción con la facultad, grado de satisfacción con la presentación de las carreras, grado de satisfacción con la carrera que más les interesó y el nivel de recomendación de la facultad y de la carrera. Por su parte, la rúbrica evalúa la comprensión de las metas de la carrera, motivación para inscribirse en la carrera, infraestructura física de la facultad, personal docente capacitado, y actividades de formación complementarias, becas y apoyos económicos complementarios.

Un aspecto por mencionar de estos instrumentos es que fueron sujetos de análisis de sus propiedades psicométricas (Mata-López et al., 2019). Específicamente se analizó la validez de facie o aparente y la validez de contenido; la primera permite la verificación de las preguntas 0 ítems que pertenecen a un fenómeno y su relevancia, así como la forma en que fueron redactados y si son comprensibles (Buela-Casal y Sierra, 1997); la segunda tiene el objetivo de establecer los aspectos relevantes del dominio del fenómeno, considerando ítems no incluidos o para eliminar los que se considere que no son relevantes (Mendoza-Mendoza y Garza, 2009). Sin embargo, un aspecto fundamental en el análisis de las propiedades psicométricas de un instrumento es el análisis de validez de constructo, la cual es referida como el principal tipo de validez (Pérez-Gil et al., 2000). En este orden, esta propiedad se define como la verificación de que la estructura del instrumento reproduce realmente la del constructo planteado (Lagunes-Córdoba, 2017).

Acorde a la significación de la propiedad psicométrica referida, el objetivo del presente trabajo fue efectuar el análisis de validez de constructo de los instrumentos mencionados, y de esta forma verificar si la estructura del instrumento reproduce realmente la del constructo planteado. Todo ello puede aportar a la orientación profesional, porque los eventos de promoción de la oferta educativa para que las instituciones de educación superior apoyen al estudiante a tomar una decisión razonada sobre la elección de una carrera. Por lo que contar con herramientas para evaluar estos eventos permitirían mejorar el proceso de promoción y calidad de la información proporcionada. 


\section{Metodología}

\section{Tipo de estudio}

Se efectuó un estudio instrumental, el cual de acuerdo con Montero y León (2007) así como Ato et al. (2013) consiste en el desarrollo de pruebas, incluyendo su diseño y adaptación, así como el estudio de sus propiedades psicométricas. De manera específica, en el presente estudio se abordó el análisis de las propiedades de validez de constructo y confiabilidad.

\section{Participantes}

Como se indicó, las JP se programan con anticipación y se invitan a las preparatorias de la misma universidad, afiliadas y externas.

Los estudiantes de preparatoria seleccionan para visitar la escuela o facultad que ofrezca la carrera de su interés. La mayoría de los bachilleratos llevan a sus estudiantes en grupo acompañados por profesores, algunos otros estudiantes llegan por sus propios medios.

Los estudiantes al finalizar el evento solicitan un comprobante de asistencia y se registran para contabilizarlos. La asistencia a estos eventos, particularmente en escuelas de ingeniería, por su naturaleza, son menos concurridas que otras áreas disciplinares, aún con las actividades de promoción realizadas, la asistencia es escasa.

A manera de ilustrar lo anterior se indica que en los eventos previos se tuvo como asistencia total para todas las carreras de la facultad 151 en 2016, 165 en 2017, y 105 en 2018, que es el año en que se realizó el presente estudio.

Los instrumentos se aplicaron al finalizar tanto la presentación como el recorrido por las instalaciones a los interesados en la carrera de Ingeniería en Sistemas Computacionales (ISC), la cual es objeto de estudio por tener problemas de matrícula y correr el riesgo de no ser pertinente, pese a la demanda creciente de profesionales en el área.

A este respecto, el cuestionario lo contestaron 74 estudiantes, mientras que la rúbrica socioformativa la contestaron 65 estudiantes (Tablas 1 y 2 ). 


\section{Tabla 1}

Datos Sociodemográficos del grupo de estudiantes que contestó el cuestionario y la rúbrica analítica socioformativa

\begin{tabular}{lll}
\hline \multicolumn{1}{c}{ Características } & Estudiantes cuestionario $(\mathbf{n}=\mathbf{7 4})$ & \multicolumn{1}{c}{ Estudiantes rúbrica (n=65) } \\
\hline Sexo: & $82 \%$ hombres & $81.54 \%$ hombres \\
& $18 \%$ mujeres & $18.46 \%$ mujeres \\
Promedio de edad en años: & 17.62 años & 17.65 años \\
Promedio de calificación del & 8.82 & 8.84 \\
último semestre de preparatoria: & $1.35 \%$ Bilingüe & $1.54 \%$ Bilingüe \\
Carrera técnica de bachillerato: & $2.70 \%$ Administración & $3.08 \%$ Administración \\
& $5.41 \%$ Dibujo & $4.62 \%$ Dibujo \\
& $6.76 \%$ No contestó & $6.15 \%$ Contabilidad \\
& $6.76 \%$ Analista Químico & $7.69 \%$ No contestó \\
& $6.76 \%$ Contabilidad & $7.69 \%$ Analista Químico \\
& $28.38 \%$ General & $32.31 \%$ Analista Programador \\
& $41.89 \%$ Analista Programador & $36.92 \%$ General \\
\hline
\end{tabular}

Fuente: elaboración propia

\section{Tabla 2}

Datos Sociodemográficos del grupo de estudiantes que contestó el cuestionario y la rúbrica analítica socioformativa (Continuación)

\begin{tabular}{lll}
\hline \multicolumn{1}{c}{ Características } & \multicolumn{1}{c}{ Estudiantes cuestionario (n=74) } & \multicolumn{1}{c}{ Estudiantes rúbrica (n=65) } \\
\hline Tipo de bachillerato: & $100 \%$ Público & $100 \%$ Público \\
& $0 \%$ Privado & $0 \%$ Privado \\
Condiciones económicas: & $1.35 \%$ Excelentes & $1.35 \%$ Excelentes \\
& $66.22 \%$ Aceptables & $66.22 \%$ Aceptables \\
& $25.68 \%$ Buenas & $25.68 \%$ Buenas \\
& $6.76 \%$ Bajas & $6.76 \%$ Bajas \\
Procedencia: & $97.30 \%$ Oficial (UCol) & $96.92 \%$ Oficial (UCol) \\
Carrera de interés: & $2.70 \%$ Otra & $3.08 \%$ Otra \\
& $85.14 \%$ Ingeniería en Software & $86.15 \%$ Ingeniería en Software \\
& $14.86 \%$ Ingeniería en Telemática & $13.85 \%$ Ingeniería en Telemática \\
\hline
\end{tabular}

Fuente: elaboración propia

Se destaca que el número total de estudiantes interesados en la carrera que asistieron fue de 90, por lo que el tamaño de ambas muestras se encuentra bajo los parámetros del 99\% de nivel de confianza y un margen de error del $10 \%$. 


\section{Instrumentos y Procedimiento}

El estudio se llevó a cabo mediante la selección de instrumentos, aplicación de estos a los asistentes al evento, y el análisis de validez de constructo y confiabilidad. Los instrumentos seleccionados corresponden a un cuestionario y una rúbrica analítica socioformativa diseñados por Mata-López et al. (2019). El primer instrumento es un cuestionario para la "Encuesta de la Jornada Profesiográfica", e integra 26 ítems en cinco dimensiones (intereses personales, grado de satisfacción de la facultad, grado de satisfacción con la presentación de las carreras, grado de satisfacción con la carrera que más me interesó, y recomendación de la facultad y de las carreras). Las preguntas fueron abiertas y de selección múltiple con cinco opciones de respuesta: nivel de satisfacción muy alto, medio, aceptable, bajo y muy bajo.

El segundo instrumento se denomina "Rúbrica de la Exposición de la Carrera" y su construcción se basó en una rúbrica socioformativa integrada por seis ítems (comprensión de las metas de la carrera, motivación para inscribirse en la carrera, infraestructura física de la facultad, personal docente capacitado, y actividades de formación complementarias, becas y apoyos económicos complementarios). En cada uno de ellos se establecieron cinco niveles de desempeño de acuerdo con la taxonomía socioformativa (Tobón, 2015), los cuales son: 1) preformal, 2) receptivo, 3) resolutivo, 4) autónomo y 5) estratégico. Para responder a un ítem se selecciona una sola de estas opciones.

Estos instrumentos fueron diseñados para evaluar la carrera de Ingeniería en Sistemas Computacionales (ISC) de la UCol, sin embargo, son tan generales que pueden ser utilizados para evaluar ferias profesiográficas (FP) y exposiciones de carreras (EC) de cualquier área de profesionalización. Se decidió orientarlos a las carreras relacionadas con la computación ya que es una de las áreas donde se han detectado problemas de matrícula, por lo que la promoción a través de eventos o actividades para proporcionar información de las carreras se convierte en una opción para incrementar la demanda. Ambos instrumentos fueron revisados por expertos, evaluados mediante juicio de expertos para analizar la validez de contenido y finalmente aplicados a grupos pilotos para determinar la confiabilidad. En este sentido, los expertos aprobaron los instrumentos, el juicio de expertos determinó la validez de contenido de ambos instrumentos y en el pilotaje la confiabilidad fue adecuada (Alfa de Cronbach: 0.92 y 0.71 respectivamente) (MataLópez et al., 2019).

\section{Análisis de validez de constructo y confiabilidad}

De manera inicial se analizó la correlación ítem-escala y se consideró que si algún ítem presentaba un valor menor a 0.20 o bien mayor a 0.95, era sujeto de eliminación (Kline, 1986; Kline, 2011). Así mismo se realizó el cálculo del coeficiente Alfa de Cronbach de manera global y acorde al mismo se verificó si la eliminación de un ítem incrementaba el valor del coeficiente. Una vez hecho este análisis, se realizó el análisis de validez de constructo mediante el Análisis Factorial Exploratorio (AFE). La elección de este método se basó en que se desea identificar la estructura factorial subyacente a los ítems (Lloret-Segura et al., 2014) y observar la correspondencia de la estructura factorial resultante respecto a la propuesta teórica. Se consultó lo indicado por Hair et al. (1999), Costello y Osborne (2005) y Mavrou (2015) referente a la muestra mínima para efectuar esta técnica estadística. Para verificar la pertinencia de los datos para este análisis se realizó a través del valor de la determinante de la matriz de correlaciones, la prueba de Kaiser Meyer Olkin y de Barlett (Pérez y Medrano, 2010; Hefetz y Liberman, 2017; Juárez-Hernández, 2018). A este respecto, los parámetros de pertinencia para efectuar el AFE fue 
que el valor de la determinante fuera cercano a cero, que en el índice de KMO fuera superior a 0.70 y finalmente que la prueba de esfericidad de Bartlett presentara una $p<0.05$ (Costello y Osborne, 2005; Pérez y Medrano, 2010; Hefetz y Liberman, 2017; Juárez-Hernández, 2018).

Posterior a verificar estos supuestos, se procedió con el análisis y se consideraron medidas en función del tamaño de muestra. De estas fue la elección del método de extracción de factorización de ejes principales, debido a que el método es robusto con muestras pequeñas y violación de normalidad (De Winter y Dodou, 2012; Hefetz y Liberman, 2017; Juárez-Hernández, 2018). El número de factores a retener se basó en la regla de Gutman-Kaiser y porcentaje de varianza explicada (50\%) (Merenda, 1997). Otra consideración tomada fue la significancia de la carga factorial respecto al tamaño de muestra, para lo cual se consultó lo indicado por Hair et al. (1999). De manera específica para el cuestionario se empleó un valor de carga factorial mayor a 0.60 y para la rúbrica mayor de 0.70 . Finalmente, se realizó el análisis de confiabilidad mediante el coeficiente Alfa de Cronbach (Cronbach, 1951), indicando que para el valor obtenido se tendrán en cuenta los criterios indicados por Nunnaly y Berstein (1994) y Taber (2018).

\section{Resultados}

Análisis de validez de constructo y confiabilidad de los cuestionarios de la "Encuesta de la Jornada Profesiográfica"

Los ítems 1-6 pertenecientes a la dimensión "Intereses personales", fueron sujetos de eliminación ya que presentaron una correlación ítem-test menor a lo indicado (0.20), consistentemente la eliminación de estos ítems incrementaba el Alfa de Cronbach que se estimó inicialmente (0.817) (Tabla 3). La prueba Kaiser Meyer Olkin (KMO: 0.839) y esfericidad de Bartlett $\left(X^{2}\right.$ : $908.835 \mathrm{Gl}$ : $231 ; p<0.001)$ denotaron la pertinencia de los datos para ser analizados mediante el AFE.

Propiamente, el AFE en su primera matriz mostró la representación de la mayoría de los ítems dentro del modelo factorial, únicamente los ítems 2, 9, 12, 14 y 21 presentaron una baja comunalidad (Tabla 3).

El análisis mostró diferencias referentes a la propuesta teórica, ya que se presentó la conformación de tres factores, los cuales poseen un autovalor mayor a uno y que en conjunto explicaron más del $57 \%$ de la varianza, denotando que el factor uno explicó más del $41 \%$, el factor dos más del $9 \%$ y el factor tres más del $7 \%$.

Al efectuar el análisis de la matriz factorial se encontró complejidad factorial en los ítems 23 , 24 y 25, por lo que se rotó la matriz, permitiendo clarificar las cargas y eliminar la complejidad factorial presentada para los ítems mencionados. Acorde a la significancia de las cargas factoriales establecidas ( $C F>0.60)$ únicamente 14 de los 21 ítems estuvieron representados en los tres factores referidos (Tabla 4). 


\section{Tabla 3}

Resultados de los análisis de correlación ítem-test y confiabilidad del cuestionario

\begin{tabular}{|c|c|c|c|}
\hline Dimensión & Ítem & $\begin{array}{l}\text { Correlación } \\
\text { ítem-test }\end{array}$ & $\begin{array}{l}\text { Alfa de } \\
\text { Cronbach }\end{array}$ \\
\hline \multirow[t]{6}{*}{$\begin{array}{l}\text { 1. Intereses } \\
\text { personales }\end{array}$} & $\begin{array}{l}\text { 1. Describe cuáles son tus principales intereses en la } \\
\text { actualidad }\end{array}$ & 0.115 & 0.820 \\
\hline & 2. Tengo claridad de la carrera que deseo cursar & 0.215 & 0.816 \\
\hline & $\begin{array}{l}\text { 3. Lo más difícil a lo que me he enfrentado para decidir } \\
\text { qué carrera estudiar es }\end{array}$ & 0.088 & 0.833 \\
\hline & $\begin{array}{l}\text { 4. Aunque todavía no hayas decidido una carrera en } \\
\text { particular, ¿qué te gustaría estudiar en la Universidad? }\end{array}$ & -0.054 & 0.824 \\
\hline & $\begin{array}{l}\text { 5. ¿Cómo te gustaría que fuera la carrera universitaria } \\
\text { que elijas? }\end{array}$ & -0.168 & 0.851 \\
\hline & $\begin{array}{l}\text { 6. ¿Cuál es el aspecto más determinante para que elijas } \\
\text { una carrera universitaria? }\end{array}$ & 0.057 & 0.842 \\
\hline \multirow{8}{*}{$\begin{array}{l}\text { 2. Grado de } \\
\text { satisfacción con la } \\
\text { facultad }\end{array}$} & $\begin{array}{l}\text { 7. ¿Cuál es el grado de satisfacción con la infraestructura } \\
\text { de la facultad? }\end{array}$ & 0.606 & 0.801 \\
\hline & $\begin{array}{l}\text { 8. ¿Cuál es el grado de satisfacción con el prestigio de la } \\
\text { facultad? }\end{array}$ & 0.633 & 0.801 \\
\hline & $\begin{array}{l}\text { 9. ¿Cuál es el grado de satisfacción con la ubicación de } \\
\text { la facultad? }\end{array}$ & 0.348 & 0.811 \\
\hline & $\begin{array}{l}\text { 10. ¿Cuál es el grado de satisfacción con el } \\
\text { reconocimiento social de la facultad? }\end{array}$ & 0.519 & 0.805 \\
\hline & $\begin{array}{l}\text { 11. ¿Cuál es el grado de satisfacción con la imagen que } \\
\text { proyecta la facultad? }\end{array}$ & 0.629 & 0.802 \\
\hline & $\begin{array}{l}\text { 12. ¿Cuál es el grado de satisfacción con las } \\
\text { posibilidades de obtener una beca? }\end{array}$ & 0.444 & 0.807 \\
\hline & $\begin{array}{l}\text { 13. ¿Cuál es el grado de satisfacción con los costos de la } \\
\text { matrícula? }\end{array}$ & 0.345 & 0.811 \\
\hline & $\begin{array}{l}\text { 14. ¿Cuál es el grado de satisfacción con el ambiente } \\
\text { que se vive en la facultad? }\end{array}$ & 0.513 & 0.806 \\
\hline \multirow{3}{*}{$\begin{array}{l}\text { 3. Grado de } \\
\text { satisfacción con la } \\
\text { presentación de } \\
\text { las carreras }\end{array}$} & $\begin{array}{l}\text { 15. Amabilidad de los profesores que me compartieron } \\
\text { los programas educativos }\end{array}$ & 0.749 & 0.801 \\
\hline & $\begin{array}{l}\text { 16. Respuestas a mis dudas por los profesores que nos } \\
\text { compartieron los programas educativos }\end{array}$ & 0.718 & 0.801 \\
\hline & 17. Proyectos presentados por los estudiantes & 0.535 & 0.803 \\
\hline \multirow{6}{*}{$\begin{array}{l}\text { 4. Grado de } \\
\text { satisfacción con la } \\
\text { carrera que más } \\
\text { me interesó }\end{array}$} & 18. Prestigio de los egresados de la carrera & 0.602 & 0.803 \\
\hline & $\begin{array}{l}\text { 19. Preparación de los docentes con nivel de maestría o } \\
\text { doctorado }\end{array}$ & 0.702 & 0.801 \\
\hline & 20. La imagen que proyecta la facultad & 0.699 & 0.800 \\
\hline & 21. Instalaciones deportivas & 0.462 & 0.805 \\
\hline & 22. Laboratorios y talleres que me mostraron & 0.633 & 0.801 \\
\hline & 23. Programas de intercambio académico & 0.530 & 0.805 \\
\hline \multirow{3}{*}{$\begin{array}{l}\text { 5. Recomendación } \\
\text { de la FIME y de la } \\
\text { carrera }\end{array}$} & $\begin{array}{l}\text { 24. Grado en el cual recomendaría a un amigo o familiar } \\
\text { la carrera que me gustaría estudiar en la facultad }\end{array}$ & 0.458 & 0.808 \\
\hline & $\begin{array}{l}\text { 25. Grado en el cual recomendaría a la facultad a un } \\
\text { amigo o familiar }\end{array}$ & 0.561 & 0.806 \\
\hline & $\begin{array}{l}\text { 26. Es probable que seleccione la facultad para estudiar } \\
\text { la carrera que me interesó }\end{array}$ & 0.543 & 0.805 \\
\hline
\end{tabular}

Fuente: elaboración propia 


\section{Tabla 4}

Comunalidades y cargas factoriales del cuestionario

\begin{tabular}{|c|c|c|c|c|c|}
\hline Dimensión & Ítem & Comunalidad & $\begin{array}{c}\text { Factor } \\
1\end{array}$ & $\begin{array}{l}\text { Factor } \\
2\end{array}$ & $\begin{array}{c}\text { Factor } \\
3\end{array}$ \\
\hline $\begin{array}{l}\text { 1. Intereses } \\
\text { personales }\end{array}$ & $\begin{array}{l}\text { 2. Tengo claridad de la carrera que deseo } \\
\text { cursar }\end{array}$ & 0.291 & . & . & . \\
\hline \multirow{8}{*}{$\begin{array}{l}\text { 2. Grado de } \\
\text { satisfacción con } \\
\text { la facultad }\end{array}$} & $\begin{array}{l}\text { 7. ¿Cuál es el grado de satisfacción con la } \\
\text { infraestructura de la facultad? }\end{array}$ & 0.577 & . & 0.679 & . \\
\hline & $\begin{array}{l}\text { 8. ¿Cuál es el grado de satisfacción con el } \\
\text { prestigio de la facultad? }\end{array}$ & 0.597 & . & 0.718 & . \\
\hline & $\begin{array}{l}\text { 9. ¿Cuál es el grado de satisfacción con la } \\
\text { ubicación de la facultad? }\end{array}$ & 0.166 & & & . \\
\hline & $\begin{array}{l}\text { 10. ¿Cuál es el grado de satisfacción con el } \\
\text { reconocimiento social de la facultad? }\end{array}$ & 0.575 & . & 0.771 & . \\
\hline & $\begin{array}{l}\text { 11. ¿Cuál es el grado de satisfacción con la } \\
\text { imagen que proyecta la facultad? }\end{array}$ & 0.596 & . & 0.757 & . \\
\hline & $\begin{array}{l}\text { 12. ¿Cuál es el grado de satisfacción con las } \\
\text { posibilidades de obtener una beca? }\end{array}$ & 0.387 & . & . & . \\
\hline & $\begin{array}{l}\text { 13. ¿Cuál es el grado de satisfacción con los } \\
\text { costos de la matrícula? }\end{array}$ & 0.443 & . & 0.771 & . \\
\hline & $\begin{array}{l}\text { 14. ¿Cuál es el grado de satisfacción con el } \\
\text { ambiente que se vive en la facultad? }\end{array}$ & 0.305 & & & . \\
\hline \multirow{3}{*}{$\begin{array}{l}\text { 3. Grado de } \\
\text { satisfacción con } \\
\text { la presentación } \\
\text { de las carreras }\end{array}$} & $\begin{array}{l}\text { 15. Amabilidad de los profesores que me } \\
\text { compartieron los programas educativos }\end{array}$ & 0.729 & 0.809 & . & . \\
\hline & $\begin{array}{l}\text { 16. Respuestas a mis dudas por los } \\
\text { profesores que nos compartieron los } \\
\text { programas educativos }\end{array}$ & 0.726 & 0.872 & . & . \\
\hline & 17. Proyectos presentados por los estudiantes & 0.427 & 0.683 & . & . \\
\hline \multirow{6}{*}{$\begin{array}{l}\text { 4. Grado de } \\
\text { satisfacción con } \\
\text { la carrera que } \\
\text { más me } \\
\text { interesó }\end{array}$} & 18. Prestigio de los egresados de la carrera & 0.546 & 0.752 & . & . \\
\hline & $\begin{array}{l}\text { 19. Preparación de los docentes con nivel de } \\
\text { maestría o doctorado }\end{array}$ & 0.632 & 0.633 & . & . \\
\hline & 20. La imagen que proyecta la facultad & 0.619 & & . & . \\
\hline & 21. Instalaciones deportivas & 0.287 & & . & . \\
\hline & 22. Laboratorios y talleres que me mostraron & 0.621 & 0.745 & . & . \\
\hline & 23. Programas de intercambio académico & 0.392 & & . & . \\
\hline \multirow{3}{*}{$\begin{array}{l}5 . \\
\text { Recomendación } \\
\text { de la FIME y de } \\
\text { la carrera }\end{array}$} & $\begin{array}{l}\text { 24. Grado en el cual recomendaría a un } \\
\text { amigo o familiar la carrera que me gustaría } \\
\text { estudiar en la facultad }\end{array}$ & 0.496 & . & . & 0.671 \\
\hline & $\begin{array}{l}\text { 25. Grado en el cual recomendaría a la } \\
\text { facultad a un amigo o familiar }\end{array}$ & 0.673 & . & . & 0.781 \\
\hline & $\begin{array}{l}\text { 26. Es probable que seleccione la facultad } \\
\text { para estudiar la carrera que me interesó }\end{array}$ & 0.572 & & . & 0.69 \\
\hline
\end{tabular}

Fuente: elaboración propia

En este sentido, el factor uno se nombró como "Grado de satisfacción con las características de la carrera" y conjuntó ítems de la dimensión "Grado de satisfacción con la presentación de las carreras" (15, 16 y 17) e ítems de la dimensión "Grado de satisfacción con la carrera que más me interesó" (18. 19 y 22). Por su parte el factor dos se denominó "Grado de satisfacción con la facultad" y se destaca que integró cinco ítems $(7,8,10,11$ y 13) de los ocho que inicialmente se habían propuesto. Finalmente, el factor tres se denominó "Recomendación de la FIME y de la carrera" e integró los tres ítems que inicialmente se propusieron en esta dimensión. La confiabilidad de la versión final del instrumento (14 ítems) mostró un valor óptimo (Alfa de Cronbach: 0.907$)$, y aceptables por factor $(0.849,0.885,0.803$ respectivamente). 
Análisis de validez de constructo y confiabilidad de la "Rúbrica de la Exposición de la Carrera"

Acorde a la correlación ítem-test ningún ítem presentó un valor menor a lo indicado (0.20) (Tabla 5); sin embargo, se indica que la eliminación del ítem seis incrementaba el valor del coeficiente de confiabilidad inicial (Alfa de Cronbach: 0.875), por lo que dicho ítem se retiró del instrumento.

La prueba Kaiser Meyer Olkin (KMO: 0.881) y esfericidad de Bartlett ( $X^{2}: 217.520$ Gl: 15; $p<0.001$ ) reveló la pertinencia de los datos para ser analizados mediante el AFE.

Propiamente, el AFE en su primera matriz mostró la representación de todos los ítems dentro del modelo factorial (Tabla 6).

El análisis mostró la conformación de un factor, el cual explicó más del 73\% de la varianza, y en este factor se encontró la representación de los cinco ítems con una carga significativa (CF>0.70).

La confiabilidad de la versión final del instrumento (de cinco ítems) mostró un valor óptimo (Alfa de Cronbach: 0.908).

\section{Tabla 5}

Resultados de los análisis de correlación ítem-test y confiabilidad de la rúbrica

\begin{tabular}{|c|c|c|c|}
\hline Dimensión & Ítem & $\begin{array}{c}\text { Correlación } \\
\text { ítem-test }\end{array}$ & $\begin{array}{l}\text { Alfa de } \\
\text { Cronbach } \\
\text { si el ítem se } \\
\text { elimina } \\
\end{array}$ \\
\hline \multirow{6}{*}{ 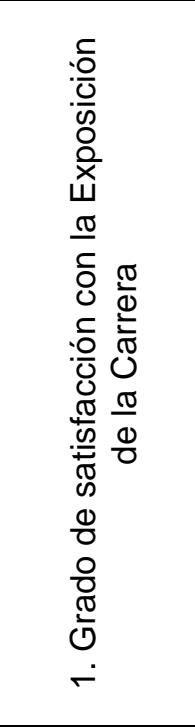 } & $\begin{array}{l}\text { 1. ¿En qué grado la exposición de las características de } \\
\text { la carrera de tu interés te permitió comprender las metas } \\
\text { de la carrera y su estructura? }\end{array}$ & 0.686 & 0.853 \\
\hline & $\begin{array}{l}\text { 2. ¿En qué grado la exposición te deja con motivación } \\
\text { para estudiar la carrera que te interesó? }\end{array}$ & 0.823 & 0.830 \\
\hline & $\begin{array}{l}\text { 3. ¿En qué grado la exposición te permitió conocer las } \\
\text { instalaciones de la facultad y te generó interés para } \\
\text { estudiar la carrera? }\end{array}$ & 0.704 & 0.851 \\
\hline & $\begin{array}{l}\text { 4. ¿En qué grado la exposición te ayudó a comprender la } \\
\text { calidad de los profesores de la carrera de tu interés? }\end{array}$ & 0.795 & 0.834 \\
\hline & $\begin{array}{l}\text { 5. ¿En qué grado la exposición te permitió conocer las } \\
\text { distintas actividades que se realizan durante los estudios } \\
\text { y te ayudó a comprender su importancia en tu formación } \\
\text { integral? }\end{array}$ & 0.738 & 0.844 \\
\hline & $\begin{array}{l}\text { 6. ¿En qué grado la exposición te permitió conocer y } \\
\text { analizar las distintas becas, movilidad y otros apoyos } \\
\text { económicos para estudiar la carrera que te interesó? }\end{array}$ & 0.446 & 0.908 \\
\hline
\end{tabular}

Fuente: elaboración propia 


\section{Tabla 6}

Comunalidades y cargas factoriales de la rúbrica

\begin{tabular}{|c|c|c|c|}
\hline Dimensión & Ítem & Comunalidad & $\begin{array}{l}\text { Carga } \\
\text { Factorial }\end{array}$ \\
\hline \multirow{5}{*}{ 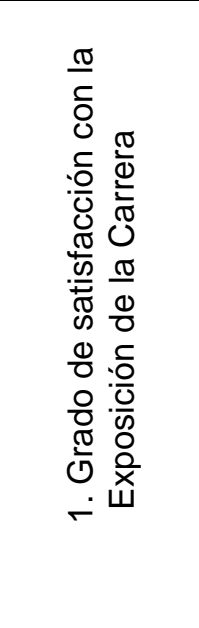 } & $\begin{array}{l}\text { 1. ¿En qué grado la exposición de las características de } \\
\text { la carrera de tu interés te permitió comprender las metas } \\
\text { de la carrera y su estructura? }\end{array}$ & 0.562 & 0.75 \\
\hline & $\begin{array}{l}\text { 2. ¿En qué grado la exposición te deja con motivación } \\
\text { para estudiar la carrera que te interesó? }\end{array}$ & 0.835 & 0.914 \\
\hline & $\begin{array}{l}\text { 3. ¿En qué grado la exposición te permitió conocer las } \\
\text { instalaciones de la facultad y te generó interés para } \\
\text { estudiar la carrera? }\end{array}$ & 0.593 & 0.77 \\
\hline & $\begin{array}{l}\text { 4. ¿En qué grado la exposición te ayudó a comprender } \\
\text { la calidad de los profesores de la carrera de tu interés? }\end{array}$ & 0.732 & 0.855 \\
\hline & $\begin{array}{l}\text { 5. ¿En qué grado la exposición te permitió conocer las } \\
\text { distintas actividades que se realizan durante los estudios } \\
\text { y te ayudó a comprender su importancia en tu formación } \\
\text { integral? }\end{array}$ & 0.615 & 0.784 \\
\hline
\end{tabular}

Fuente: elaboración propia

\section{Conclusiones y Discusión}

Las estrategias implementadas para incrementar la matrícula son relevantes, ya que el descenso de esta puede poner en riesgo la viabilidad y pertinencia de un programa educativo (Barr y Turner, 2013). Como se indicó, particularmente destacan las JP y las EC implementadas por la UCol para dar a conocer las carreras profesionales que ofrece en cada ciclo escolar, además de publicidad en redes sociales, radio y televisión universitaria. Específicamente para el área de ingeniería, la asistencia al evento es históricamente baja como se mencionó previamente, lo cual obedece a que las ingenierías ofertadas por la facultad son consideradas por voz de los mismos estudiantes como difíciles, y a pesar de que ofrecen un abanico de oportunidades laborales, por su complejidad regularmente prefieren otras opciones. Por lo anterior hay que destacar la importancia de que los estudiantes conozcan esta amplia gama de oportunidades de trabajo, así como desmitificar que estas carreras son complejas, lo cual se puede abordar con las JP y EC para que sean funcionales para estos fines.

Otro aspecto para destacar es que no se contaba con mecanismos de evaluación para estas actividades (JP y EC), que pudieran brindar información sobre la percepción y funcionalidad para los asistentes; lo cual es útil para que la institución pueda mejorar estos eventos, por lo que la "Encuesta de la Jornada Profesiográfica" y la "Rúbrica de la Exposición de la Carrera" representan un aporte. Originalmente estos fueron diseñados para evaluar ISC por pertenecer a una de las áreas donde se han detectado problemas en la cantidad de aspirantes; sin embargo, al no incluir en los ítems aspectos técnicos o específicos de la carrera, dichos instrumentos pueden ser utilizados en cualquier disciplina; razón por la cual la realización de eventos informativos para la promoción de las carreras es una estrategia adecuada para incrementar la demanda. 
Por otro lado, si bien los instrumentos mencionados fueron en una primera fase validados en términos de facie y contenido (Mata-López et al., 2019), permitiendo verificar la pertinencia, representatividad y relevancia de los elementos que en ellos se suscriben (dimensiones e ítems) al constructo objetivo (Buela-Casal y Sierra, 1997; Meliá, 2001; Haynes et al., 1995; Koller et al., 2017), se requiere el análisis de validez de constructo para comprobar que la estructura del instrumento reproduzca realmente el constructo planteado (Lagunes-Córdoba, 2017), y verificar hasta qué punto los ítems que conforman un instrumento tienen una representación adecuada (Mavrou, 2015).

A este respecto, el análisis de esta propiedad en el presente trabajo se efectuó mediante el AFE, que es una de las técnicas más utilizadas para la validación del constructo de instrumentos de evaluación (Pérez y Medrano, 2010). La elección del AFE fue determinada con el objetivo de identificar la estructura factorial subyacente a los ítems (Lloret-Segura et al., 2014) y observar la correspondencia de la estructura factorial respecto a la propuesta teórica. Para la aplicación de esta técnica, se consideró que el análisis es factible efectuarlo con tamaños muestrales mayores a 50 casos (Mavrou, 2015), sin embargo, acorde al tamaño de muestra en el presente, se consideraron una serie de criterios y restricciones como fue la elección de un método de extracción adecuado (factorización de ejes principales), ya que es robusto con tamaños de muestra pequeños (De Winter y Dodou, 2012) y el empleo de relación tamaño de muestra y magnitud de la carga factorial, denotada como significancia (Hair et al., 1999). Respecto al uso de la significación de la carga factorial en relación con el tamaño de muestra, se especifica que brinda una precisión del 95\% en los intervalos de confianza y una potencia estadística del $80 \%$.

Para la "Encuesta de la Jornada Profesiográfica", el análisis reveló diferencias ya que, de las cinco dimensiones propuestas, únicamente se denotaron tres (Grado de satisfacción con las características de la carrera, Grado de satisfacción con la facultad y Recomendación de la FIME y de la carrera). En este sentido, para la dimensión uno "Intereses Personales", en su mayoría los ítems no cumplieron con el criterio de correlación ítem-test, por lo que no se consideraron para el AFE y el ítem que cumplió "Tengo claridad de la carrera que deseo cursar", no presentó una adecuada comunalidad y su carga factorial no fue significativa. Probablemente lo anterior se deba a que las preguntas indagan en lo que les interesa a los estudiantes, y no precisamente evalúan la percepción de estos acerca de las JP y la EC. Los estudiantes apenas están iniciando el proceso de definir el área que les interesa y están en la etapa de identificar carreras, intereses, escuelas, universidades, etc. Los resultados denotan que posiblemente estas preguntas requieran un proceso de maduración y conforme vayan adquiriendo más información van a estar en mejores condiciones de contestarlas.

De manera específica, el factor uno se denominó "Grado de satisfacción con las características de la carrera" e integró ítems de las dimensiones "Grado de satisfacción con la presentación de las carreras" y "Grado de satisfacción con la carrera que más me interesó". En este sentido la unión de estas dos dimensiones se debe a que los ítems abordan aspectos relativos a profesores, estudiantes, egresados y su ambiente educativo, los cuales son relevantes puesto que se ajustan al propósito del instrumento, el cual es conocer qué información proporcionada durante la JP es de interés para los estudiantes. En este sentido, el papel de los docentes es importante (ítems 15, 16, 19), estudiantes (ítem 17), egresados (ítem 18) e infraestructura de laboratorios y talleres (ítem 22). Estos ítems son importantes pues reflejan claramente que a los estudiantes les interesa de los profesores su preparación y la relación que pueden llegar a tener con ellos durante sus estudios, de los estudiantes los tipos de proyectos que realizan, de los egresados su prestigio, y de la facultad los laboratorios y talleres disponibles.

De esta dimensión también es importante denotar que los ítems referentes a la imagen que proyecta la facultad (ítem 20), instalaciones deportivas (ítem 21) y programas de intercambio académico (ítem 23), no presentaron una carga factorial significativa. En este sentido, el ítem 21, originalmente fue considerado de acuerdo con García-Alcaraz y Moreno-Morales (2012), que manifiestan la importancia que muchos alumnos le dan a las actividades deportivas organizadas por la institución. Acorde a los resultados del presente estudio, como se indicó, este ítem no 
revelaría la importancia ya que el principal interés de los asistentes está en conocer aspectos relacionados con la carrera y las instalaciones destinadas a ella. El ítem correspondiente a la imagen proyectada por la facultad en relación con la carrera se tomó en cuenta porque las organizaciones consideran que la imagen es un aspecto valioso (Casanoves-Boix et al., 2018), sin embargo, este ítem probablemente no está representado porque al parecer a los estudiantes les interesa más la imagen de la facultad que de la carrera, pues el ítem correspondiente a la imagen de la facultad sí estuvo representado. Del mismo modo, el ítem correspondiente a los programas de intercambio académico se consideró debido a la importancia actual de la movilidad académica, ya que esta puede incidir en el futuro de los proyectos personales de los estudiantes además de su inserción laboral (Izquierdo-Rus y Farías-Gragmena, 2018; Rodríguez-Martínez et al., 2019; Passarini et al., 2019). La falta de representación puede deberse al desconocimiento de los aspirantes de estos programas y porque en sus intereses inmediatos está el escoger una carrera antes de pensar a dónde irse de intercambio.

Referente al factor dos, se especifica que se correspondió con la dimensión propuesta "Grado de satisfacción con la facultad". Los ítems que estuvieron representados abordan aspectos importantes: imagen, prestigio, reconocimiento social, infraestructura y costos de la matrícula. Si una universidad logra establecer su prestigio y reputación puede mejorar su imagen con respecto a sus estudiantes, además de que les proporciona reconocimiento social (Casanoves-Boix et al., 2017). Con respecto a la infraestructura, se considera que esta tiene un impacto positivo y significativo en el logro académico (Melo-Becerra et al., 2017). El costo de la matrícula revela su importancia porque influye en las decisiones de los estudiantes, y que incluso lo consideran más importante que aspectos académicos (García-Alcaraz y Moreno-Morales, 2012; Fondevila-Gascón y del-Olmo-Arriaga, 2013). Por otro lado, los ítems de esta dimensión que abordan la ubicación, ambiente de la facultad y las posibilidades para obtener una beca no están representados. La ubicación es una característica particular de la facultad donde se encuentra la carrera objeto de estudio, y al estar alejada de la ciudad, simplemente por el hecho de asistir a las actividades de promoción puede ser indicativo de que no es un aspecto relevante para los aspirantes, además de que, en el estado la oferta académica pública en computación/informática está limitada a tres instituciones educativas principalmente. Por otro lado, al ser la gran mayoría egresados de bachillerato de la UCol, el ambiente puede ser un aspecto no tan importante, pues la mayoría ya lo conocen e incluso hay campus que tienen distintos niveles académicos y hay mucha convivencia entre ellos. Por último, al saber que la UCol tiene una gran variedad de apoyos económicos, puede ser la razón por la que obtener una beca no sea lo más importante, pues sabe que existen grandes posibilidades de obtenerla.

Finalmente, el factor tres se correspondió con la dimensión propuesta "Recomendación de la FIME y de la carrera" aborda el grado en el que los estudiantes recomendarían la carrera y la facultad, así como la probabilidad de que las seleccionen para continuar con sus estudios. Este aspecto es importante puesto que en algunas ocasiones los estudiantes eligen la carrera precisamente por recomendaciones de amigos (Cordón-Lagares et al., 2013; Gómez, 2014). En este sentido, los ítems propuestos se encuentran representados, especificándose su importancia y denotando la trascendencia de esta.

Acorde a estos resultados se puede indicar que este nuevo arreglo es consistente con lo indicado como objetivos funcionales del AFE, el cual permite reinterpretar las variables, agrupando y excluyendo ítems brindando una mayor coherencia al instrumento (Faúndez et al., 2018). Por lo anterior, se puede considerar que los ítems conservados son los que poseen representación adecuada al constructo a evaluar (Mavrou, 2015).

Para la rúbrica, los resultados son consistentes con lo estipulado teóricamente; sin embargo, se destaca lo relacionado con el ítem seis que no fue incluido porque afectaba el valor de la confiabilidad. Este ítem específicamente evalúa el grado en que la exposición permitió conocer y analizar las distintas becas, movilidad y otros apoyos económicos para estudiar la carrera que le interesó al estudiante. Este ítem se incluyó debido a que los apoyos económicos son considerados como relevantes en la permanencia de los estudiantes (Velázquez-Narváez y 
González-Medina, 2017). Probablemente los resultados obtenidos se pueden deber a que este tema fue el último abordado durante la EC y no tuvo el impacto que se pretendía, o que por la duración de la presentación no se le dio el énfasis necesario como para que quedara en la memoria del colectivo estudiantil.

Referente a la confiabilidad, para ambos instrumentos se presentaron valores óptimos (Nunnally y Bernstein, 1994; Taber, 2018), lo cual refiere la precisión del instrumento, correlación entre ítems y representación del concepto abordado (Haynes et al., 1995; Welch y Comer, 1988). Por supuesto que, dado el tamaño de la muestra, estos cálculos deben tomarse con reserva, ya que el coeficiente tiende a ser inestable con muestras pequeñas (Charter, 2003).

A partir de estos resultados, se puede concluir que el análisis efectuado favoreció la depuración de los instrumentos, sin embargo, la limitación del presente estudio fue el tamaño de muestra debido a que en las carreras de ingeniería se presenta una baja asistencia de estudiantes a estos eventos, por lo que los resultados no pueden ser considerados concluyentes. Acorde a esto, se motiva a continuar con la aplicación a una muestra mayor para contrastar los resultados obtenidos en el presente. Por último, es importante recalcar que, pese a que los instrumentos se aplicaron a los estudiantes interesados en estudiar una carrera de computación/informática, estos pueden aplicarse a cualquier área profesional, pues no son preguntas especializadas específicamente en un área de formación.

Las jornadas profesiográficas y exposición de carrera son actividades relevantes dentro del campo de la orientación profesional, ya que brindan al estudiante información de la oferta educativa y elementos para una decisión razonada sobre la elección de carrera. Por lo tanto, este estudio aporta dos instrumentos con adecuadas propiedades psicométricas, mediante los cuales se podrá obtener evidencia válida y confiable sobre el cumplimiento de las metas de estas actividades.

\section{Referencias bibliográficas}

Aguilar-Salinas, W. E., De Las Fuentes Lara, M., Rivera Castellón, R. E. y Íñiguez-Monroy, C. G. (2018). Perfiles de estudiantes asociados a las características de reprobación de las asignaturas de ciencias básicas en ingeniería. Revista Boletín Redipe, 7(8), 129-145. https://revista.redipe.org/index.php/1/article/view/548

Álvarez-Lires, F. J., Arias-Correa, A., Serrallé-Marzoa, J. F. y Varela-Losada, M. (2014). Elección de estudios de ingeniería: Influencia de la educación científica y de los estereotipos de género en la autoestima de las alumnas. Revista de investigación en educación, 12(1), 5472. http://hdl.handle.net/20.500.12799/3266

Aničić, K. P., Divjak, B. y Arbanas, K. (2017). Preparing ICT Graduates for Real-World Challenges: Results of a Meta-Analysis. IEEE Transactions on Education, 60, 191-197. https://doi.org/10.1109/TE.2016.2633959

Arroyo-Almaguer, M., Pérez-Pizano, S. E., Rodríguez-Vargas, M.J., Rico-Moreno, J.L., RamosLópez, H. y Ambriz-Colín, F. (2019). Programa Integral Extracurricular que fortalece la pertinencia de los Programas Educativos en TIC y que atienden a diversos criterios de las categorías de evaluación con fines de acreditación. Tecnología Educativa, Revista CONAIC, 6(2), 57-67. https://terc.mx/index.php/terc/article/view/38 
Arroyo-Hernández, J., Gamboa-Araya, R. y Hidalgo-Mora, R. (2012). Proyecto divulgación y promoción de la carrera de bachillerato y licenciatura en la enseñanza de la matemática en zonas rurales de Costa Rica: alcances y resultados. Uniciencia, 26(1-2), 125-133. https://www.redalyc.org/articulo.oa?id=475947764012

Ato, M., López-García, J. y Benavente, A. (2013). A classification system for research designs in psychology. Annals of Psychology, 29(3), 1038-1059.

https://doi.org/10.6018/analesps.29.3.178511

Barbón-Pérez, O. G. y Fernández-Pino, J. W. (2018). Rol de la gestión educativa estratégica en la gestión del conocimiento, la ciencia, la tecnología y la innovación en la educación superior. Educación Médica, 19(1), 51-55. https://doi.org/10.1016/j.edumed.2016.12.001

Barr, A. y Turner, S. E. (2013). Expanding Enrollments and Contracting State Budgets: The Effect of the Great Recession on Higher Education. The ANNALS of the American Academy of Political and Social Science, 650(1), 168-193. https://doi.org/10.1177/0002716213500035

Bravo-Torres, G. y Vergara-Tamayo, M. A. (2018). Factores que determinan la elección de carrera profesional en estudiantes de undécimo grado de colegios públicos y privados de Barrancabermeja. Psicoespacios: Revista virtual de la Institución Universitaria de Envigado, 12(20), 35-48. http://bibliotecadigital.iue.edu.co/jspui/handle/20.500.12717/2279

Brito, J. G., Laaser, W. y Toloza, E. A. (2015). El uso de redes sociales por parte de las universidades a nivel institucional. Un estudio comparativo. Revista De Educación a Distancia, 32, 1-38. https://revistas.um.es/red/article/view/23307

Buela-Casal, G. y Sierra, J. C. (1997). Manual de evaluación psicológica: fundamentos, técnicas y aplicaciones. Siglo XXI.

Byrne, J. R., O'sullivan, K. y Sullivan, K. (2017). An IoT and Wearable Technology Hackathon for Promoting Careers in Computer Science. IEEE Transactions on Education, 60(1), 50-58. https://doi.org/10.1109/TE.2016.2626252

Canek, R., Chicas, Y. y Rodas, O. (2019). Fomenting STEM Careers through Robotics Competitions: A Work in Progress. 2019 IEEE Integrated STEM Education Conference (ISEC), 270-273. https://doi.org/10.1109/ISECon.2019.8882058

Casanoves-Boix, J., Küster-Boluda, I. y Vila-López, N. (2017). La lealtad del estudiante como clave para construir marca universitaria. ESIC Market Economics and Business Journal, 48(3), 553-578. https://doi.org/10.7200/esicm.158.0483.3e

Casanoves-Boix, J., Küster-Boluda, I. y Vila-López, N. (2018). ¿Por qué las instituciones de educación superior deben apostar por la marca? Revista de Investigación Educativa, 37(1), 111-127. https://doi.org/10.6018/rie.37.1.291191

Charter, R. A. (2003). Study samples are too small to produce sufficiently precise reliability coefficients. The Journal of General Psychology, 130(2), 117-129. https://doi.org/10.1080/00221300309601280

Cordón-Lagares, A., Cordón-Lagares, E. y García-Ordaz, F. (2013). Evidencias empíricas en el proceso de elección de los estudios universitarios. Enseñanza \& Teaching: Revista $\begin{array}{llll}\text { Interuniversitaria de } \quad \text { Didáctica, } & \text { 30(2), }\end{array}$ https://dialnet.unirioja.es/servlet/articulo?codigo=4091427

Costello, A. B. y Osborne, J. W. (2005). Best practices in exploratory factor analysis: Four recommendations for getting the most from your analysis. Practical Assessment, Research, \& Evaluation, 10(7), 1-9. https://dx.doi.org/10.4135/9781412995627.d8 
Cronbach, L. J. (1951). Coefficient alpha and the internal structure of tests. Psychometrika, 16(3), 297-334. https://doi.org/10.1007/BF02310555

De Vega, F. F., García, M. C. y Chávez, F. (2017). Involving local administrations in STEM promotion: How to extend STEM initiatives to a whole region. 2017 IEEE Frontiers in Education Conference, 1-7. https://doi.org/10.1109/FIE.2017.8190726

De Winter, J. C. F. y Dodou, D. (2012). Factor recovery by principal axis factoring and maximum likelihood factor analysis as a function of factor pattern and sample size. Journal of Applied Statistics, 39(4), 695-710. https://doi.org/10.1080/02664763.2011.610445

Díaz-Izaguirre, Y., Cruz-Cruz, C. y Suárez-Garay, A. (2019). Reafirmación profesional en los estudiantes: desafío para los docentes de la educación superior. Edumecentro, 11(3), 4560. http://www.revedumecentro.sld.cu/index.php/edumc/article/view/1395

Duran, S. E. (2014). Promoción de las instituciones educativas bajo el contexto de la interculturalidad. Formación Gerencial, 13(2), 297-316.

Faúndez, V. O., Monte, P. G., Espinoza, R. M. M., Capot, R. B., Collarte, D. F. y Ferraz, H. F. (2018). Validez factorial del cuestionario para la evaluación del síndrome de quemarse por el trabajo en profesionales de servicios. Interciencia: Revista de ciencia y tecnología de América, 43(6), 417-424. http://dx.doi.org/10.6035/AgoraSalut.2015.1.32

Fondevila-Gascón, J. F. y Del-Olmo-Arriaga, J. L. (2013). Factores de elección de carreras de Ciencias de la Comunicación en España: el peso de la ética. Estudios sobre el Mensaje Periodístico, 19, 735-744. https://doi.org/10.5209/rev ESMP.2013.v19.42156

Fuentes-Navarro, M. T. (2010). La orientación profesional para elegir fundamentadamente una ocupación: Propuesta alternativa. Revista Mexicana de Psicología, 27(2), 237-246. https://www.redalyc.org/articulo.oa?id=243016324012

García-Alcaraz, J. L. y Moreno-Morales, C. S. (2012). Factores considerados al seleccionar una universidad. Caso Ciudad Juárez. Revista Mexicana de Investigación Educativa, 17(52), 287-305. https://www.redalyc.org/articulo.oa?id=14023076012

Gómez, S. M. (2014). Estudio exploratorio en estudiantes universitarios. Referencias sociodemográficas y elección de carrera. Praxis Educativa (Arg), 18(1), 50-57. https://www.redalyc.org/articulo.oa?id=153137899005

González, E. I. (2015). Factores de contexto socioeconómicos y educativos en estudiantes de nivel superior, sugerencia para una realidad actual. Interamerican Journal of Psychology, 49(3), 399-412. https://www.redalyc.org/articulo.oa?id=28446020010

Hair, J. F., Anderson, R. E., Tatham, R. L. y Black, W. C. (1999). Análisis multivariante. Prentice Hall.

Haynes, S., Richard, D. y Kubany, E. (1995). Content Validity in Psychological Assessment: A Funtional Approach to Concepts and Methods. Psychological Assessment, 7(3), 238-247. https://doi.org/10.1037//1040-3590.7.3.238

Hefetz, A. y Liberman, G. (2017). The factor analysis procedure for exploration: a short guide with examples. Culture and Education, 29(3), 526-562. https://doi.org/10.1080/11356405.2017.1365425

Hernández-Salamanca, O. (2020). Percepción social de la orientación escolar en orientadores de Bogotá. REOP - Revista Española de Orientación y Psicopedagogía, 31(1), 131-144. https://doi.org/10.5944/reop.vol.31.num.1.2020.27294 
Izquierdo-Rus, T. y Farías-Gragmena, A. (2018). Empleabilidad y expectativa de logro en la inserción laboral de los estudiantes universitarios. Revista Española de Orientación y Psicopedagogía, 29(2), 29-40. https://doi.org/10.5944/reop.vol.29.num.2.2018.23151

Juárez-Hernández, L. G. (2018). Manual práctico de estadística básica para la investigación. Kresearch.

Kline, P. (1986). A handbook of test construction: Introduction to psychometric design. Methuen.

Kline, R. B. (2011). Principles and practice of structural equation modeling. Guilford Press.

Koller, I., Levenson, M. R. y Glück, J. (2017). What do you think you are measuring? A mixedmethods procedure for assessing the content validity of test items and theory-based scaling. Frontiers in psychology, 8, 1-20. https://doi.org/10.3389/fpsyg.2017.00126

Lagunes-Córdoba, R. (2017). Recomendaciones sobre los procedimientos de construcción y validación de instrumentos y escalas de medición en la psicología de la salud. Psicología y Salud, 27(1), 5-18. https://psicologiaysalud.uv.mx/index.php/psicysalud/article/view/2431

Lara-Barrón, A. y Valadez-Díaz, D. (2011). Factores que afectan la trayectoria escolar de egresadas(os) de enfermería. Revista de Enfermería del Instituto Mexicano del Seguro Social, 19(3), 143-148.

https://www.medigraphic.com/cgi-bin/new/resumen.cgi?IDARTICULO=31772

Lloret-Segura, S., Ferreres-Traver, A., Hernández-Baeza, A. y Tomás-Marco, I. (2014). El análisis factorial exploratorio de los ítems: una guía práctica, revisada y actualizada. Anales de Psicología, 30(3), 1151-1169. https://www.redalyc.org/articulo.oa?id=16731690031

Manzano-Sánchez, H., Mulford-Ramírez, E. y Upegui, P. (2019). Aspiraciones profesionales y universitarias de estudiantes de grado once de bachillerato de una institución educativa pública de Cali/Professional and college aspirations among 11 th grade high school students in Cali. REOP - Revista Española de Orientación y Psicopedagogía, 30(3), 10-25. https://doi.org/10.5944/reop.vol.30.num.3.2019.26270

Mata-López, W. A., Juárez-Hernández, L. G., Tobón, S. y Montesinos-López, O. A. (2019). Percepción de la calidad de eventos académicos para la promoción de facultades y carreras: una propuesta instrumental. Espacios, 40(9), 1-24. https://www.revistaespacios.com/a19v40n09/19400924.html

Mata-López, W. A. y Tobón, S. (2019). Analysis of Factors Associated to the Enrollment and Demand of Computing-Related Careers. Social Sciences, 8, 1-18. https://doi.org/10.3390/socsci8010001

Mavrou, I. (2015). Análisis factorial exploratorio: cuestiones conceptuales y metodológicas. Revista Nebrija de Lingüística Aplicada, 19, 71-80. https://doi.org/10.26378/rnlael019283

Meliá, J. L. (2001). Teoría de la Fiabilidad y la Validez. Cristóbal Serrano.

Melo-Becerra, L. A., Ramos-Forero, J. E. y Hernández-Santamaría, P. O. (2017). La educación superior en Colombia: situación actual y análisis de eficiencia. Revista Desarrollo y Sociedad, 78, 59-111. https://www.redalyc.org/articulo.oa?id=169149895003

Mendoza-Mendoza, J. y Garza, J. B. (2009). La medición en el proceso de investigación científica: Evaluación de validez de contenido y confiabilidad. Innovaciones de negocios, 6(11), 1732. http://eprints.uanl.mx/12508/ 
Merenda, P. (1997). A guide to the proper use of Factor Analysis in the conduct and reporting of research: pitfalls to avoid. Measurement and evaluation in counseling and evaluation, 30, 156-163. https://doi.org/10.1080/07481756.1997.12068936

Montero, I. y León, O. G. (2007). Guía para nombrar los estudios de investigación en Psicología. International Journal of Clinical and Health Psychology, 7, 847-862. https://www.redalyc.org/articulo.oa?id=33770318

Nunnally, J. C. y Bernstein, I. H. (1994). Psychometric theory (3rd ed.). McGraw-Hill, Inc.

Passarini, J., Zucarelli, V., Silba-Oliva, M., Sánchez, E., De-León, F., Ojeda, N. y Theiler, J. (2019). Evaluación de la movilidad académica de grado: opinión de participantes de los Programas Escala y Marca. Integración y Conocimiento, 8(2), 41-53.

Payton, J., Barnes, T., Buch, K., Rorrer, A., Zuo, H. y Naolu, B. (2016). Promoting computing faculty success through interinstitutional Faculty Learning Communities. 2016 Research on Equity and Sustained Participation in Engineering, Computing, and Technology (RESPECT), 1-8. https://doi.org/10.1109/RESPECT.2016.7836163

Pérez, E. y Medrano, L. (2010). Análisis Factorial Exploratorio: Bases conceptuales y metodológicas. Revista Argentina de Ciencias del comportamiento, 2(1), 58-66. https://doi.org/10.32348/1852.4206.v2.n1.15924

Pérez-Gil, J., Chacón-Moscoso, S. y Moreno-Rodríguez, R. (2000). Validez de Constructo: el uso del análisis factorial exploratorio-confirmatorio para obtener evidencias de validez. Psicothema, 12(2), 442-446. http://www.psicothema.com/psicothema.asp?id=601

Quatrocchi, P., Flores, C., Cassullo, G., Moulia, L., Marco, M., Shafferstein C., Pereda, Y. y Siniuk, D. (2017). Motivación y género en la elección de la carrera. Revista de Educación y Desarrollo, 41, 27-35. https://www.cucs.udg.mx/revistas/edu desarrollo/anteriores/41/41 Casullo.pdf

Retana-Alvarado, D. A. y Vázquez-Bernal, B. (2016). Ferias de Ciencia y Tecnología de Costa Rica: una experiencia que motiva la elección de carreras científicas y tecnológicas. Campo Abierto, 35(1), 13-30.

https://mascvuex.unex.es/revistas/index.php/campoabierto/article/view/2824

Rodríguez, J., Butt, S. y Fredericks, T. (2014). Pre-college activities to promote positive perception of engineering and engineering technology careers. 2014 International Conference on Interactive Collaborative Learning, 715-719. https://doi.org/10.1109/ICL.2014.7017858

Rodríguez-Martínez, A., Cortés-Pascual, A. y Val-Blasco, S. (2019). Análisis de la mejora del nivel de empleabilidad de los universitarios mediante la mejora de competencias transversales y habilidades. Revista Española de Orientación y Psicopedagogía, 30(3), 102-119. https://doi.org/10.5944/reop.vol.30.num.3.2019.26275

Romero-Rodríguez, S., Figuera-Gazo, P., Freixa-Niella, M. y Llanes-Ordóñez, J. (2019). Adaptabilidad de la Carrera en estudiantes universitarios: Un estudio a través de entrevistas autobiográficas. Revista de Investigación Educativa, 37(2), 379-394. https://doi.org/10.6018/rie.37.2.322441

Taber, K. S. (2018). The use of Cronbach's alpha when developing and reporting research instruments in science education. Research in Science Education, 48(6), 1273-1296. https://doi.org/10.1007/s11165-016-9602-2

Tobón, S. (2015). Evaluación socioformativa: estrategias e instrumentos. CIFE. 
Velázquez-Narváez, Y. y González-Medina, M. A. (2017). Factores asociados a la permanencia de estudiantes universitarios: caso UAMM-UAT. Revista de la Educación Superior, 46(184), 117-138. https://doi.org/10.1016/j.resu.2017.11.003

Villada, C. A., López, L. M., Patiño, C. D., Ramírez, W., Jurado, C. M. y Ossa, J. (2002). Factores Asociados a la Elección de Carrera y Universidad. Uni-pluri/versidad, 2(2), 23-30. Welch, S. y Comer, J. (1988). Quantitative Methods for Public Administration. Brooks/Cole Publishing.

Zafra-Tristancho, S., Martínez-Lozano, J. y Vergel-Ortega, M. (2014). Indicadores para evaluar la pertinencia social en la oferta académica de programas. Revista Logos Ciencia y Tecnología, 6(1), 142-155. https://doi.org/10.22335/rlct.v6i1.361

Zaharakis, I. D., Sklavos, N. y Kameas, A. (2016). Exploiting Ubiquitous Computing, Mobile Computing and the Internet of Things to Promote Science Education. 2016 8th IFIP International Conference on New Technologies, Mobility and Security (NTMS), 1-2. https://doi.org/10.1109/NTMS.2016.7792451

Zapata, G. y Tejeda, I. (2016). Publicidad engañosa en educación superior: definiciones, regulación y una revisión de la publicidad en prensa escrita. Calidad en la educación, 44, 197-242. http://dx.doi.org/10.4067/S0718-45652016000100008

\section{Anexos}

\section{Anexo 1. Encuesta de la Jornada Profesiográfica}

\section{Dimensión}

Grado de satisfacción con las características de la carrera

Grado de satisfacción con la facultad
1. Amabilidad de los profesores que me compartieron los programas educativos

2. Respuestas a mis dudas por los profesores que nos compartieron los programas educativos

3. Proyectos presentados por los estudiantes

4. Prestigio de los egresados de la carrera

5. Preparación de los docentes con nivel de maestría o doctorado

6 . Laboratorios y talleres que me mostraron

7. ¿Cuál es el grado de satisfacción con la infraestructura de la facultad?

8. ¿Cuál es el grado de satisfacción con el prestigio de la facultad?

9. ¿Cuál es el grado de satisfacción con el reconocimiento social de la facultad?

10. ¿Cuál es el grado de satisfacción con la imagen que proyecta la facultad?

11. ¿Cuál es el grado de satisfacción con los costos de la matrícula? 
Recomendación de la FIME y de la carrera
12. Grado en el cual recomendaría a un amigo o familiar la carrera que me gustaría estudiar en la facultad

13. Grado en el cual recomendaría a la facultad a un amigo o familiar 14. Es probable que seleccione la facultad para estudiar la carrera que me interesó

\title{
Anexo 2. Rúbrica de la Exposición de la Carrera
}

\author{
Preguntas \\ Niveles socioformativos de desempeño: \\ a) Preformal, b) Receptivo, c) Resolutivo, d) Autónomo y e) Estratégico
}

1. ¿En qué grado la exposición de las características de la carrera en Ingeniería en Sistemas Computacionales te permitió comprender las metas de la carrera y su estructura?

a) No comprendí las metas de la carrera en Ingeniería que me interesó.

b) Identifiqué en la exposición las metas de la carrera que me interesó, pero no las relaciono con el futuro profesional.

c) Comprendí las metas de la carrera y las puedo diferenciar.

d) A partir de la exposición pude analizar las metas de la carrera, su estructura y áreas.

e) La exposición me permitió vincular la carrera con los retos del futuro, la considero muy relevante para la sociedad y mi proyección profesional y laboral.

2. ¿En qué grado la exposición te deja con motivación para estudiar la carrera en Ingeniería en Sistemas Computacionales?

a) La exposición no logró motivarme para estudiar la Ingeniería que me interesó.

b) En la exposición logré identificar algunas ventajas de estudiar la carrera, pero no me motivan a estudiarla.

c) La exposición me ayudó a comprender con elementos concretos y ejemplos las ventajas de la carrera, pero aún no me siento con motivación para inscribirme

d) La exposición me ayudó a comprender la importancia de la carrera para mi futuro profesional y probablemente me inscribiré.

e) La exposición me dejó con gran motivación y con seguridad me inscribiré en la carrera porque se ajusta a lo que quiero hacer en el campo laboral.

3. ¿En qué grado la exposición te permitió conocer las instalaciones de la FIME y te generó interés para estudiar Ingeniería en Sistemas Computacionales?

a) No distinguí durante la exposición los distintos tipos de instalaciones con que cuenta la Facultad para el desarrollo de la carrera.

b) La exposición describió las instalaciones deportivas, laboratorios, talleres y áreas comunes que tiene la Facultad, pero no me parecieron interesantes.

c) Con la exposición conocí las instalaciones deportivas, los talleres y laboratorios; considero que son adecuadas para el desarrollo de la carrera.

d) Con la exposición conocí la infraestructura física que tiene la Facultad y me permitió reflexionar y valorar las ventajas y oportunidades que voy a tener si estudio la carrera que me interesó.

e) La exposición me generó interés para estudiar la carrera que me interesó debido a las instalaciones deportivas, talleres y laboratorios que ponen a disposición durante los estudios de la carrera.

4. ¿En qué grado la exposición te ayudó a comprender la calidad de los profesores de la carrera de Ingeniería en Sistemas Computacionales?

a) En la exposición no quedo clara la importancia de los docentes y del personal administrativo para la Facultad.

b) En la exposición se mencionó que tanto los profesores como el personal administrativo de la 
Facultad están bien capacitados.

c) La exposición me permitió comprender que la mayor parte del personal académico tiene estudios de posgrado, además de que el personal administrativo está bien preparado para darnos la atención debida.

d) La exposición me permitió comprender el valor agregado de la carrera que me interesó, al contar con profesores con maestría y doctorado, lo que me va a permitir adquirir una mejor preparación académica.

e) La exposición me generó interés y compromiso para estudiar la carrera que me interesó, ya que la preparación académica de los profesores con maestría y doctorado les va a permitir enseñarnos mejor.

5. ¿En qué grado la exposición te permitió conocer las distintas actividades que se realizan durante los estudios y te ayudó a comprender su importancia en tu formación integral?

a) Con la exposición no me quedaron muy claras las actividades complementarias que se realizan en la Facultad.

b) La exposición me ayudó a identificar algunas de las actividades complementarias que se realizan durante los estudios de la carrera que me interesó en la Facultad.

c) Con la exposición comprendí la importancia de las actividades académicas, deportivas, sociales y culturales que se planifican durante el desarrollo de los estudios de la carrera que me interesó.

d) La exposición me ayudó a entender que las actividades académicas, deportivas, sociales y culturales realizadas durante los estudios de la carrera, están integradas de tal forma que mejoran la calidad de sus egresados.

e) La exposición hizo que me interesara estudiar la carrera, debido a que las actividades de formación complementarias están encaminadas a mejorar sustancialmente la formación de los egresados.

Fecha de entrada: 21 abril 2020

Fecha de revisión: 29 junio 2020

Fecha de aceptación: 23 octubre 2020 